\title{
Feature extraction and artificial neural networks for the on-the-fly classification of high-dimensional thermochemical spaces in adaptive-chemistry simulations - ERRATUM
}

\author{
Giuseppe D'Alessio, Alberto Cuoci and Alessandro Parente
}

doi: 10.1017/dce.2021.2 Published online by Cambridge University Press; $12^{\text {th }}$ April 2021

Keywords: Adaptive chemistry; artificial neural networks; feature extraction; local principal component analysis; ERRATUM

During the typesetting process, errors were introduced into Equations 13 and 14. The publishers apologise for this error that does not impact the results of the paper. The correct version of the equations are:

$$
\begin{gathered}
\sigma_{i}(\mathbf{z})=\frac{e^{z_{i}}}{\sum_{j=1}^{k} e^{z_{j}}} \\
t=\frac{\sum_{i=1}^{q} \lambda_{i}}{\sum_{j=1}^{p} \lambda_{j}}
\end{gathered}
$$

\section{Reference}

D’Alessio, G., Cuoci, A., \& Parente, A. (2021). Feature extraction and artificial neural networks for the on-the-fly classification of high-dimensional thermochemical spaces in adaptive-chemistry simulations. Data-Centric Engineering, 2, E2. doi:10.1017/ dce. 2021.2

Cite this article: D'Alessio G, Cuoci A and Parente A (2021). Feature extraction and artificial neural networks for the on-the-fly classification of high-dimensional thermochemical spaces in adaptive-chemistry simulations - ERRATUM. Data-Centric Engineering, 2: e4. doi:10.1017/dce.2021.4 